\title{
Assessment of Physico-chemical Properties of Soil in Dadrol Block, Shahjahanpur District, Uttar Pradesh, India
}

\author{
Mohd Noman*, Arun Alfred David, Tarence Thomas, \\ Narendra Swaroop and Amreen Hasan
}

Department of Soil Science and Agricultural Chemistry, (Naini Agricultural Institute), Sam

Higginbottom University of Agricultural, Technology and Sciences, Prayagraj-211 007, Uttar

Pradesh, India

*Corresponding author

\section{A B S T R A C T}

\section{Keywords}

Soil Physicochemical properties, depth, Nutrients, etc

Article Info

\section{Accepted:}

12 June 2021

Available Online:

10 July 2021
Industrial activities sequel a demolishing influence on the soil health. The present investigation was analysed at Sam Higginbottom University of agriculture technology and sciences in the department of Soil Science and Agricultural Chemistry lab. The objective of this study was to analyse various soil physico-chemical properties. Depth wise soil samples were collected from three selected farmer's field in Piprola village of Dadrol block of Shahjahanpur district. Three different sites were taken in each farmer's field represented three profile depths viz., 0-15, 15-30 and 30-45 cm, totally 27 samples were collected. The results revealed that the texture was sandy loam, bulk density ranged from 1 to $1.23 \mathrm{Mg} \mathrm{m}^{-3}$, particle density from 2 to $2.66 \mathrm{Mg} \mathrm{m}^{-3}$, pore space from 42.52 to $55.19(\%)$, water holding capacity from 54.54 to $78.21(\%)$ and specific gravity from 2.1 to 4.43 . The $\mathrm{pH}$ ranged from7.17 to 8.84 , E.C. from 0.15 to $0.51\left(\mathrm{dS} \mathrm{m}^{-1}\right)$.Available nitrogen ranged from 142.16 to $424.39\left(\mathrm{~kg} \mathrm{ha}^{-1}\right)$, phosphorous from 20.67 to $41.42\left(\mathrm{~kg} \mathrm{ha}^{-1}\right)$ and potassium from 62.88 to $108.23\left(\mathrm{~kg} \mathrm{ha}^{-1}\right)$. Exchangeable calcium, magnesium ranged from 0.7 to $2.57,0.2$ to $0.82\left(\mathrm{cmol}\left(\mathrm{p}^{+}\right) \mathrm{kg}^{-}\right.$ ${ }^{1}$ ) and available sulphur from 6.03 to 11.34 (ppm). Overall soils were in moderate condition. Farmers required maintaining soil health card, adopting suitable management practices and providing proper nutrition to the soil to overcome the pollution effect.

\section{Introduction}

Agriculture is one of the world's oldest economic practices. It has developed into a technologically advanced industry and it currently plays a substantial role in global sustainability(Harrell, 2014) ${ }^{(7)}$. Soils need maintenance, but exploitation of soils has only intensified due to increasing pressure. Today, soil, crops and vegetables globally provide ample food for 7 billion people. The availability though is unevenly distributed and 1 billion people are structurally underfed. To provide for food for 9-10 billion people by 
2050 , the biophysical as well as the socioeconomic availability of food as well as of the food productive capacity are to be strongly improved. Crucial is the capacity of land users worldwide to manage their soils sustainably and productively (ISRIC, 2021) ${ }^{(10)}$. Soil health is the "state of the soil being in sound physical, chemical, and biological condition, having the capability to sustain the growth and development of land plants" (Idowu et al., 2019) ${ }^{(9)}$. Soil acts as a filter and buffer for contaminants, but its potential to cope is finite. If the capacity of the soil to mitigate the effects of pollutants is exceeded, the contaminants pollute other compartments of the environment. Soil pollution causes a chain reaction that starts with reduced soil biodiversity, alters organic matter incorporation rates, and then weakens soil structure and ability to resist erosion. Main sources of soil pollution are by industrial activities that release large amounts of chemicals into the environment during manufacturing, transportation and use (Bhattacharyya et al., 2015) ${ }^{(2)}$. Degradation of the soil is the most problematic problem of the Indian soil. The process of soil degradation is the result of natural forces and human activities. India is now reaping what it had sown decades ago (Supriya, 2021) ${ }^{(23)}$. The food productivity and environmental quality is dependent on the physico-chemical properties of soil, so it is very important to know the basic knowledge about the physico-chemical properties of soil (Tale et al., 2015) ${ }^{(24)}$.

\section{Materials and Methods}

The present study entitled "Assessment of Physico-chemical Properties of Soil in Dadrol Block, Shahjahanpur District, Uttar Pradesh, India", was carried out during 2020-21 and comprised of a lab experiment which was carried out in Department of Soil Science and Agricultural chemistry, Naini Agricultural Institute, Sam Higginbottom University of
Agriculture Technology and Sciences, Prayagraj (U.P.)., India.

\section{Site details}

Shahjahanpur is situated in west-central part of Uttar Pradesh. It is an Agriculture based district of Uttar Pradesh lying between latitude $27^{\circ} 27^{\prime} \mathrm{N}$ and $28^{\circ} 28^{\prime} \mathrm{N}$, and longitude $79^{\circ} 19^{\prime} \mathrm{E}$ and $80^{\circ} 23^{\prime} \mathrm{E}$. Its geographical area is 4,575 $\mathrm{km}^{2}\left(1,766 \mathrm{~m}^{2}\right)$ and at the altitude of 194 metres (600 feet) above the sea level. Shahjahanpur district has the population of 3006538 as per census data of 2011. Dadrol block population is 168586 . Geographical area of Piprola Ahmedpur village is 408.55 ha with the population of 5000 as per census data of 2011, in which male population is 2660 and female population is 2340 . The soils in the district are deep and well drained, with loamy surface. Wheat is grown in the maximum area of the district, followed by rice. Other crops of minor importance are sugarcane, potato, cotton etc. There are many Industrial units in the district, namely wood/wooden based furniture's; paper \& paper products; leather based; chemical/chemical based; rubber, plastic \& petro based; metal based (Steel fabrication); Soda water; cotton textile etc.(Ranjan, 2013) ${ }^{(17)}$.

\section{Soil sampling}

Soil samples were collected from village Piprola Ahmedpur in Shahjahanpur district. In the village 3 farmers was selected randomly for sampling. Soil samples were collected from each farmer's field after harvest or before sowing. Three different sites were taken in each farmer's field represented three profile depths viz., 0-15 cm, 15-30 cm and 30$45 \mathrm{~cm}$, totally 27 samples were collected with 9 samples representing one farmer's field. At sampling site, soil samples were collected separately by a random selection from field with help of khurpi, spade, digging bar and 
meter scale. Samples were collected from centre of the fields in order to avoid the edge effect. Each soil sample is about 500mg collected from the $0-15 \mathrm{~cm}$ layer (which represented the plough layer), $15-30 \mathrm{~cm}$ and $30-45 \mathrm{~cm}$ depth.

\section{Analysis of physico-chemical parameters}

Soil textural analysis of particles less than 2 mm was performed by the hydrometer method (Bouyoucos, 1927) (4). The bulk density, particle density, pore space and water holding capacity was determined by the graduated 100 $\mathrm{ml}$ measuring cylinder method (Muthuvel et al., 1992) ${ }^{(14)}$. Specific gravity of soil was determined by the relative density bottle or pycnometer method as laid out by Black $(1965)^{(3)}$. The $\mathrm{pH}$ was determined by1:2 soilwater suspension method using digital $\mathrm{pH}$ meter (Jackson, 1958) ${ }^{(11)}$. EC was determined by1:2 soil-water suspension method using digital EC meter (Wilcox, 1950) ${ }^{(29)}$. Organic carbon was determined by the wet oxidation method (Walkley and Black, 1947) ${ }^{(27)}$. Available $\mathrm{N}$ was determined by alkaline potassium permanganate method (Subbiah and Asija, 1956) ${ }^{(21)}$. Available $\mathrm{P}$ was determined by colorimetric method (Olsen et al., 1954) (16). Available K was determined by flame photometer method (Toth and Prince, 1949) (25). Exchangeable calcium and magnesium was determined by neutral ammonium acetate extraction method or EDTA method (Cheng and Bray, 1951) (5). Available $S$ was determined by turbidimetric method (Bardsley and Lancaster, 1960) ${ }^{(1)}$.

\section{Statistical analysis}

The data recorded during the course of investigation was subjected to statistical analysis by the method of analysis of variance (ANOVA) technique (Fisher, 1960) ${ }^{(6)}$. The type of ANOVA adopted for the experiment was two-factor analysis without replication.
The implemented design of experiment in the analysis done was Completely Randomized Design (CRD). It is used when experimental units are homogenous as it involves only two basic principles of the design of experiment, viz., replication and randomization. CRD is used for laboratory purpose only. The significant and non-significant treatment effects were judged on the basis of ' $F$ ' (variance ratio) test.

\section{Results and Discussion}

The soil texture in Piprola Ahmedpur was found to be dominantly sandy loam. The sand, silt and clay (\%) content were $77.27 \%, 8.16$ $\%$ and $14.57 \%$ respectively. Most of the crops are grown in these soils because they retain more water and nutrients. Similar finding were reported by Khadka et al., (2017) ${ }^{(12)}$. As presented in table 2 and fig. 1 statistical accumulation on bulk density $\left(\mathrm{Mg} \mathrm{m}^{-3}\right)$ of soil in village Piprola Ahmedpur. No significant difference was found due to depth and significant difference was found due to site. The bulk density ranged from 1.00 to 1.23 $\left(\mathrm{Mg} \mathrm{m}{ }^{-3}\right)$. The maximum value found in $\mathrm{F}_{3} \mathrm{~S}_{2}\left(30-45 \mathrm{~cm}\right.$ depth) $1.23\left(\mathrm{Mg} \mathrm{m}^{-3}\right)$ and the minimum value found in $\mathrm{F}_{2} \mathrm{~S}_{1}(0-15 \mathrm{~cm}$ depth)1.00( $\left.\mathrm{Mg} \mathrm{\textrm {m } ^ { - 3 }}\right)$. The bulk density increases with the increase in soil depth. The reason is that soil compactness will be more at high depth and soil organic carbon will be decreased with increasing the depth. Similar finding were reported by Singh et al., (2020) ${ }^{(20)}$. As depicted in table 2 and fig. 2 statistical accumulation on particle density $\left(\mathrm{Mg} \mathrm{m}^{-3}\right)$ of soil in village Piprola Ahmedpur. No significant difference was found due to depth and no significant difference was found due to site. The particle density ranged from 2.00 to $2.66\left(\mathrm{Mg} \mathrm{m}^{-3}\right)$. The maximum value found in $\mathrm{F}_{1} \mathrm{~S}_{1}(0-15 \mathrm{~cm}$ depth $) 2.66\left(\mathrm{Mg} \mathrm{m}^{-3}\right)$ which indicates that the soil has comparatively lower organic matter and the minimum value found in $\mathrm{F}_{2} \mathrm{~S}_{2}\left(0-15 \mathrm{~cm}\right.$ depth) $2.00\left(\mathrm{Mg} \mathrm{m}^{-3}\right)$ which 
indicates the presence of high organic matter. Particle density varies according to the mineral content of the soil particles. Similar finding were reported by Yuvarani et al., (2019) ${ }^{(30)}$.As shown in table 2 and fig. 3 statistical accumulation on pore space \% of soil in village Piprola Ahmedpur. Significant difference was found due to depth and significant difference was found due to site. The pore space \% ranged from 42.52 to $55.19 \%$. The maximum value found in $\mathrm{F}_{2} \mathrm{~S}_{1}(0-$ $15 \mathrm{~cm}$ depth) $55.19 \%$ and the minimum value found in $\mathrm{F}_{3} \mathrm{~S}_{2}(30-45 \mathrm{~cm}$ depth) $42.52 \%$. Pore space was found to decrease with increase in depth attributed to increase in compaction in the sub surface. Surface soils are having high amount of macro and micro pores compared to sub surface soils due to presence of high organic matter. Similar finding were reported by Verma et al., (2019) ${ }^{(26)}$. As presented in table 3 and fig. 4 statistical accumulation on water holding capacity (\%) of soil in village Piprola Ahmedpur. Significant difference was found due to depth and no significant difference was found due to site. The water holding capacity (\%) ranged from54.54 to $78.21 \%$. The maximum value found in $\mathrm{F}_{1} \mathrm{~S}_{3}(0-15 \mathrm{~cm}$ depth) $78.21 \%$ and the minimum value found in $\mathrm{F}_{3} \mathrm{~S}_{2}(15-30 \mathrm{~cm}$ depth) $54.54 \%$. WHC value decreases with the increasing depth because of soil compaction and reduction in pore space.

Similar finding were reported by Singh et al., $(2019)^{(18)}$.As depicted in table 3 and fig. 5 statistical accumulation on specific gravity of soil in village Piprola Ahmedpur. No significant difference was found due to depth and no significant difference was found due to site. The specific gravity ranged from 2.04 to 2.45. The maximum value found in $\mathrm{F}_{2} \mathrm{~S}_{2}$ (15$30 \mathrm{~cm}$ depth) 2.45 and the minimum value found in $\mathrm{F}_{2} \mathrm{~S}_{1}(30-45 \mathrm{~cm}$ depth) 2.04 and this due to presence of organic matter and porous particles in soil. Similar finding were reported by Sujatha et al., (2016) ${ }^{(22)}$.
As presented in table 4 and fig. 6statistical accumulation on $\mathrm{pH}$ of soil in village Piprola Ahmedpur. Significant difference was found due to depth and significant difference was found due to site. The $\mathrm{pH}$ ranged from7.17 to 8.84. The maximum value found in $\mathrm{F}_{3} \mathrm{~S}_{1}$ (30$45 \mathrm{~cm}$ depth) 8.84 and the minimum value found in $\mathrm{F}_{1} \mathrm{~S}_{1}(0-15 \mathrm{~cm}$ depth) 7.17 , thereby indicating the soils are moderately alkaline. $\mathrm{pH}$ value increases with the increasing depth because the upper horizons receive maximum leaching by rainfall and by dissolved carbonic acids and presence of high amount of exchangeable sodium ions. Similar finding were reported by Okolo et al., (2016) ${ }^{(15)}$. As depicted in table 4 and fig. 7statistical accumulation on EC $\left(\mathrm{dS} \mathrm{m}^{-1}\right)$ of soil in village Piprola Ahmedpur. Significant difference was found due to depth and significant difference was found due to site. The electrical conductivity ranged from 0.15 to $0.51 \mathrm{dS} \mathrm{m}^{-1}$. The maximum value found in $\mathrm{F}_{1} \mathrm{~S}_{1}(30-45 \mathrm{~cm}$ depth) $0.51 \mathrm{dS} \mathrm{m}^{-1}$ and the minimum value found in $\mathrm{F}_{3} \mathrm{~S}_{1}\left(15-30 \mathrm{~cm}\right.$ depth) $0.15 \mathrm{dS} \mathrm{m} \mathrm{m}^{-1}$. It indicates that the soil are non-saline and salinity effect is mostly negligible for the crops. Similar finding were reported by Mallick et al., (2019) ${ }^{(13)}$.As shown in table 4 and fig. 8statistical accumulation on organic carbon (\%) of soil in village Piprola Ahmedpur. Significant difference was found due to depth and significant difference was found due to site. The soil organic carbon (\%) ranged from 0.58 to $2.40(\%)$.

The maximum value found in $\mathrm{F}_{3} \mathrm{~S}_{1}(0-15 \mathrm{~cm}$ depth) $2.40(\%)$ and the minimum value found in $\mathrm{F}_{1} \mathrm{~S}_{3}(30-45 \mathrm{~cm}$ depth) $0.58(\%)$. The organic carbon decreases with increasing depth due to the fact that surface soil contains undecomposed and partial decomposed organic matter while subsoil contains decomposed organic matter which has undergone chemical and biological changes. Similar finding were reported by Singh et al., (2012) ${ }^{(19)}$. 
Table.1 Sampling sites and GPS coordinates of Dadrol block, Shahjahanpur

\begin{tabular}{|c|c|c|c|c|c|}
\hline S. No. & Farmer & Village & Site & $\begin{array}{l}\text { Latitude } \\
\left({ }^{\circ} \mathbf{N}\right)\end{array}$ & $\begin{array}{c}\text { Longitude } \\
\left({ }^{\circ} \mathbf{E}\right)\end{array}$ \\
\hline \multirow[t]{3}{*}{1} & \multirow[t]{3}{*}{ Mr. Gulgari $\left(\mathrm{F}_{1}\right)$} & \multirow[t]{3}{*}{ Piprola Ahmedpur } & $\mathrm{S}_{1}$ & $27^{\circ} 49^{\prime} 29^{\prime \prime}$ & $79^{\circ} 55^{\prime} 40^{\prime \prime}$ \\
\hline & & & $\mathrm{S}_{2}$ & $27^{\circ} 50^{\prime} 30^{\prime \prime}$ & $79^{\circ} 49^{\prime} 30^{\prime \prime}$ \\
\hline & & & $\mathrm{S}_{3}$ & $27^{\circ} 48^{\prime} 29^{\prime \prime}$ & $79^{\circ} 55^{\prime} 33^{\prime \prime}$ \\
\hline \multirow[t]{3}{*}{2} & \multirow[t]{3}{*}{ Mr. Raghunandan Prasad ( $\left.\mathrm{F}_{2}\right)$} & \multirow[t]{3}{*}{ Piprola Ahmedpur } & $\mathrm{S}_{1}$ & $27^{\circ} 50^{\prime} 30^{\prime \prime}$ & $79^{\circ} 50^{\prime} 30^{\prime \prime}$ \\
\hline & & & $\mathrm{S}_{2}$ & $27^{\circ} 50^{\prime} 30^{\prime \prime}$ & $79^{\circ} 49^{\prime} 29^{\prime \prime}$ \\
\hline & & & $\mathrm{S}_{3}$ & $27^{\circ} 51^{\prime} 28^{\prime \prime}$ & $79^{\circ} 52^{\prime} 33^{\prime \prime}$ \\
\hline \multirow[t]{3}{*}{3} & \multirow[t]{3}{*}{ Mr. Sonpal Singh $\left(\mathrm{F}_{3}\right)$} & \multirow[t]{3}{*}{ Piprola Ahmedpur } & $\mathrm{S}_{1}$ & $27^{\circ} 50^{\prime} 30^{\prime \prime}$ & $79^{\circ} 52^{\prime} 31^{\prime \prime}$ \\
\hline & & & $\mathrm{S}_{2}$ & $27^{\circ} 49^{\prime} 29^{\prime \prime}$ & $79^{\circ} 50^{\prime} 30^{\prime \prime}$ \\
\hline & & & $\mathrm{S}_{3}$ & $27^{\circ} 49^{\prime} 29^{\prime \prime}$ & $79^{\circ} 50^{\prime} 30^{\prime \prime}$ \\
\hline
\end{tabular}

Table.2 Assessment of Bulk density, Particle density and Pore spaceof Soil from different depth 0-15, 15-30 and 30-45 cm of village Piprola Ahmedpur, Shahjahanpur

\begin{tabular}{|c|c|c|c|c|c|c|c|c|c|}
\hline \multirow{2}{*}{$\begin{array}{c}\text { Farmer } \\
\text { site }\end{array}$} & \multicolumn{3}{|c|}{ Bulk density $\left(\mathrm{Mg} \mathrm{m}^{-3}\right)$} & \multicolumn{3}{|c|}{ Particle density $\left(\mathrm{Mg} \mathrm{m}^{-3}\right)$} & \multicolumn{3}{|c|}{ Pore space $(\%)$} \\
\hline & $\begin{array}{c}0-15 \\
\mathrm{~cm}\end{array}$ & $\begin{array}{c}15-30 \\
\mathrm{~cm}\end{array}$ & $30-45 \mathrm{~cm}$ & $\begin{array}{c}0-15 \\
\mathrm{~cm}\end{array}$ & $\begin{array}{c}\text { 15-30 } \\
\text { cm }\end{array}$ & $30-45 \mathrm{~cm}$ & $\begin{array}{c}0-15 \\
\text { cm }\end{array}$ & $\begin{array}{c}15-30 \\
\text { cm }\end{array}$ & $30-45 \mathrm{~cm}$ \\
\hline $\mathbf{F}_{1} \mathbf{S}_{1}$ & 1.05 & 1.11 & 1.17 & 2.66 & 2.66 & 2.50 & 53.50 & 47.23 & 43.43 \\
\hline $\mathbf{F}_{1} \mathbf{S}_{2}$ & 1.05 & 1.05 & 1.05 & 2.50 & 2.50 & 2.66 & 51.32 & 46.37 & 45.22 \\
\hline $\mathbf{F}_{\mathbf{1}} \mathbf{S}_{3}$ & 1.02 & 1.05 & 1.05 & 2.50 & 2.66 & 2.61 & 52.75 & 43.55 & 42.56 \\
\hline $\mathbf{F}_{2} \mathbf{S}_{1}$ & 1.00 & 1.05 & 1.17 & 2.65 & 2.22 & 2.65 & 55.19 & 51.39 & 47.63 \\
\hline $\mathbf{F}_{2} \mathbf{S}_{2}$ & 1.00 & 1.05 & 1.05 & 2.00 & 2.50 & 2.22 & 54.33 & 52.63 & 50.00 \\
\hline $\mathbf{F}_{2} \mathbf{S}_{3}$ & 1.00 & 1.11 & 1.11 & 2.15 & 2.22 & 2.50 & 52.68 & 50.38 & 45.27 \\
\hline $\mathbf{F}_{3} \mathbf{S}_{1}$ & 1.05 & 1.11 & 1.05 & 2.50 & 2.35 & 2.66 & 50.52 & 47.89 & 42.77 \\
\hline $\mathbf{F}_{3} \mathbf{S}_{2}$ & 1.05 & 1.11 & 1.23 & 2.50 & 2.35 & 2.66 & 47.89 & 45.77 & 42.52 \\
\hline \multirow[t]{2}{*}{$\mathbf{F}_{3} \mathbf{S}_{3}$} & 1.11 & 1.11 & 1.11 & 2.22 & 2.35 & 2.35 & 54.23 & 52.77 & 52.77 \\
\hline & $\begin{array}{l}\text { F- } \\
\text { test }\end{array}$ & $\begin{array}{c}\text { S. Ed. } \\
( \pm)\end{array}$ & $\begin{array}{l}\text { C.D. @ } \\
\text { 0.05\% }\end{array}$ & $\begin{array}{l}\text { F- } \\
\text { test }\end{array}$ & $\begin{array}{c}\text { S. Ed. } \\
( \pm)\end{array}$ & $\begin{array}{l}\text { C.D. @ } \\
\text { 0.05\% }\end{array}$ & F-test & $\begin{array}{c}\text { S. Ed. } \\
( \pm)\end{array}$ & $\begin{array}{l}\text { C.D. @ } \\
\text { O.05\% }\end{array}$ \\
\hline $\begin{array}{l}\text { Due to } \\
\text { depth }\end{array}$ & NS & 0.037118 & 0.005604 & NS & 0.0687 & 0.204737 & S & 3.358068 & 0.000002 \\
\hline $\begin{array}{l}\text { Due to } \\
\text { site }\end{array}$ & $\mathrm{S}$ & 0.033665 & 0.113281 & NS & 0.138604 & 0.066577 & S & 2.783892 & 0.000247 \\
\hline
\end{tabular}


Table.3 Assessment of Water holding capacity and Specific gravity of Soil from different depth 0-15, 15-30 and 30-45 cm of village Piprola Ahmedpur, Shahjahanpur

\begin{tabular}{|c|c|c|c|c|c|c|}
\hline \multirow[t]{2}{*}{ Farmer site } & \multicolumn{3}{|c|}{ Water holding capacity (\%) } & \multicolumn{3}{|c|}{ Specific gravity } \\
\hline & $0-15 \mathrm{~cm}$ & $15-30 \mathrm{~cm}$ & $30-45 \mathrm{~cm}$ & $0-15 \mathrm{~cm}$ & $15-30 \mathrm{~cm}$ & $30-45 \mathrm{~cm}$ \\
\hline $\mathbf{F}_{1} \mathbf{S}_{1}$ & 62.85 & 58.82 & 65.23 & 2.35 & 2.10 & 2.31 \\
\hline $\mathbf{F}_{1} \mathbf{S}_{2}$ & 61.42 & 58.91 & 64.51 & 2.08 & 1.98 & 2.43 \\
\hline $\mathbf{F}_{1} \mathbf{S}_{3}$ & 78.21 & 75.00 & 62.50 & 2.27 & 2.14 & 1.98 \\
\hline $\mathbf{F}_{2} \mathbf{S}_{1}$ & 76.66 & 64.51 & 70.58 & 2.08 & 2.07 & 2.04 \\
\hline $\mathbf{F}_{2} \mathbf{S}_{2}$ & 69.69 & 60.60 & 65.71 & 2.31 & 2.45 & 2.18 \\
\hline $\mathbf{F}_{2} \mathbf{S}_{3}$ & 62.16 & 61.79 & 63.63 & 2.38 & 2.41 & 2.08 \\
\hline $\mathbf{F}_{3} \mathbf{S}_{1}$ & 72.72 & 64.70 & 66.66 & 2.20 & 2.26 & 2.43 \\
\hline $\mathbf{F}_{3} \mathbf{S}_{2}$ & 59.37 & 54.54 & 62.82 & 2.40 & 2.23 & 2.70 \\
\hline \multirow[t]{2}{*}{$\mathbf{F}_{3} S_{3}$} & 69.69 & 70.58 & 64.7 & 2.19 & 2.34 & 2.09 \\
\hline & F-test & S. Ed. $( \pm)$ & C.D. @ $0.05 \%$ & F-test & S. Ed. $( \pm)$ & C.D. @ $0.05 \%$ \\
\hline Due to depth & $\mathrm{S}$ & 2.426042 & 0.074028 & NS & 0.017356 & 0.910851 \\
\hline Due to site & NS & 4.455908 & 0.016553 & NS & 0.113419 & 0.294745 \\
\hline
\end{tabular}

Table.4 Assessment of Soil pH, EC and organic carbonof Soil from different depth 0-15, 15-30 and 30-45 cm of village Piprola Ahmedpur, Shahjahanpur

\begin{tabular}{|c|c|c|c|c|c|c|c|c|c|}
\hline \multirow[t]{2}{*}{$\begin{array}{l}\text { Farmer } \\
\text { site }\end{array}$} & \multicolumn{3}{|c|}{ Soil pH } & \multicolumn{3}{|c|}{$\begin{array}{c}\text { Electrical conductivity (dS } \\
\text { m }^{-1} \text { ) }\end{array}$} & \multicolumn{3}{|c|}{ Soil organic carbon (\%) } \\
\hline & $\begin{array}{c}0-15 \\
\mathrm{~cm}\end{array}$ & $\begin{array}{c}15-30 \\
\text { cm }\end{array}$ & $30-45 \mathrm{~cm}$ & $\begin{array}{c}0-15 \\
\text { cm }\end{array}$ & $\begin{array}{c}15-30 \\
\mathrm{~cm}\end{array}$ & $30-45 \mathrm{~cm}$ & $\begin{array}{c}0-15 \\
\mathrm{~cm}\end{array}$ & $\begin{array}{c}15-30 \\
\text { cm }\end{array}$ & $30-45 \mathrm{~cm}$ \\
\hline $\mathbf{F}_{1} \mathbf{S}_{1}$ & 7.17 & 8.04 & 8.65 & 0.25 & 0.28 & 0.51 & 1.50 & 1.12 & 0.97 \\
\hline $\mathbf{F}_{1} \mathbf{S}_{2}$ & 7.24 & 7.65 & 7.74 & 0.29 & 0.32 & 0.34 & 1.20 & 1.13 & 1.08 \\
\hline $\mathbf{F}_{1} \mathbf{S}_{\mathbf{3}}$ & 7.89 & 8.17 & 8.23 & 0.29 & 0.33 & 0.35 & 1.10 & 0.87 & 0.58 \\
\hline $\mathbf{F}_{2} \mathbf{S}_{1}$ & 8.12 & 8.25 & 8.18 & 0.35 & 0.41 & 0.43 & 1.65 & 1.54 & 1.35 \\
\hline $\mathbf{F}_{2} \mathbf{S}_{2}$ & 8.32 & 8.40 & 8.54 & 0.29 & 0.30 & 0.28 & 1.30 & 1.21 & 1.01 \\
\hline $\mathbf{F}_{2} \mathbf{S}_{3}$ & 8.05 & 8.11 & 8.28 & 0.29 & 0.33 & 0.38 & 1.14 & 1.02 & 0.79 \\
\hline $\mathbf{F}_{3} \mathbf{S}_{1}$ & 8.54 & 8.73 & 8.84 & 0.16 & 0.15 & 0.17 & 2.40 & 2.25 & 1.80 \\
\hline $\mathbf{F}_{3} \mathbf{S}_{2}$ & 8.32 & 8.24 & 8.58 & 0.24 & 0.28 & 0.32 & 2.10 & 1.10 & 0.83 \\
\hline \multirow[t]{2}{*}{$\mathbf{F}_{3} \mathbf{S}_{3}$} & 8.54 & 8.73 & 8.79 & 0.20 & 0.24 & 0.23 & 1.11 & 1.05 & 0.82 \\
\hline & $\begin{array}{l}\text { F- } \\
\text { test }\end{array}$ & $\begin{array}{c}\text { S. Ed. } \\
( \pm)\end{array}$ & $\begin{array}{l}\text { C.D. @ } \\
\text { 0.05\% }\end{array}$ & $\begin{array}{l}\text { F- } \\
\text { test }\end{array}$ & $\begin{array}{c}\text { S. Ed. } \\
( \pm)\end{array}$ & $\begin{array}{l}\text { C.D. @ } \\
\text { 0.05\% }\end{array}$ & $\begin{array}{l}\text { F- } \\
\text { test }\end{array}$ & $\begin{array}{c}\text { S. Ed. } \\
( \pm)\end{array}$ & $\begin{array}{l}\text { C.D. @ } \\
\text { 0.05\% }\end{array}$ \\
\hline $\begin{array}{l}\text { Due to } \\
\text { depth }\end{array}$ & $S$ & 0.203198 & 0.00464 & $\mathrm{~S}$ & 0.036226 & 0.011973 & $\mathrm{~S}$ & 0.237271 & 0.000281 \\
\hline $\begin{array}{l}\text { Due to } \\
\text { site }\end{array}$ & $S$ & 0.365023 & 0.000198 & $S$ & 0.070119 & 0.000376 & $S$ & 0.388219 & 0.00001 \\
\hline
\end{tabular}


Table.5 Assessment of available Nitrogen, Phosphorous and of Soil from different depth 0-15, 15-30 and 30-45 cm of village Piprola Ahmedpur, Shahjahanpur

\begin{tabular}{|c|c|c|c|c|c|c|c|c|c|}
\hline \multirow{2}{*}{$\begin{array}{c}\text { Farmer } \\
\text { site }\end{array}$} & \multicolumn{3}{|c|}{ Nitrogen $\left(\mathrm{kg} \mathrm{ha}^{-1}\right)$} & \multicolumn{3}{|c|}{ Phosphorous $\left(\mathrm{kg} \mathrm{ha}^{-1}\right)$} & \multicolumn{3}{|c|}{ Potassium $\left(\mathrm{kg} \mathrm{ha}^{-1}\right)$} \\
\hline & $\begin{array}{c}0-15 \\
\text { cm }\end{array}$ & $\begin{array}{c}15-30 \\
\mathrm{~cm}\end{array}$ & $30-45 \mathrm{~cm}$ & $\begin{array}{c}0-15 \\
\text { cm }\end{array}$ & $\begin{array}{c}15-30 \\
\mathrm{~cm}\end{array}$ & $30-45 \mathrm{~cm}$ & $\begin{array}{c}0-15 \\
\text { cm }\end{array}$ & $\begin{array}{c}15-30 \\
\mathrm{~cm}\end{array}$ & $30-45 \mathrm{~cm}$ \\
\hline $\mathbf{F}_{1} \mathbf{S}_{1}$ & 424.39 & 330.08 & 157.18 & 41.42 & 37.54 & 25.60 & 105.33 & 97.54 & 78.07 \\
\hline $\mathbf{F}_{1} \mathbf{S}_{2}$ & 411.21 & 306.34 & 166.36 & 40.51 & 34.67 & 24.32 & 101.91 & 82.16 & 70.86 \\
\hline $\mathbf{F}_{1} \mathbf{S}_{3}$ & 406.45 & 317.88 & 173.47 & 41.03 & 35.29 & 26.71 & 102.21 & 77.21 & 67.32 \\
\hline $\mathbf{F}_{2} \mathbf{S}_{1}$ & 285.34 & 237.65 & 154.65 & 36.15 & 27.03 & 21.38 & 108.23 & 93.27 & 81.29 \\
\hline $\mathbf{F}_{2} \mathbf{S}_{2}$ & 279.77 & 221.32 & 142.16 & 34.21 & 28.18 & 22.08 & 106.29 & 88.53 & 72.81 \\
\hline $\mathbf{F}_{2} \mathbf{S}_{3}$ & 260.92 & 220.05 & 157.18 & 33.62 & 29.41 & 20.67 & 101.10 & 72.91 & 65.22 \\
\hline $\mathbf{F}_{3} \mathbf{S}_{1}$ & 323.76 & 287.65 & 185.43 & 37.90 & 32.28 & 28.79 & 103.15 & 81.04 & 63.22 \\
\hline $\mathbf{F}_{3} \mathbf{S}_{2}$ & 312.21 & 262.34 & 166.32 & 38.24 & 33.18 & 30.71 & 102.34 & 73.31 & 62.88 \\
\hline $\mathbf{F}_{3} \mathbf{S}_{3}$ & 304.93 & 260.92 & 179.18 & 38.46 & 32.81 & 32.39 & 104.45 & 78.21 & 64.39 \\
\hline & F-test & $\begin{array}{l}\text { S. Ed. } \\
( \pm)\end{array}$ & $\begin{array}{l}\text { C.D. @ } \\
\text { 0.05\% }\end{array}$ & $\begin{array}{l}\text { F- } \\
\text { test }\end{array}$ & $\begin{array}{c}\text { S. Ed. } \\
( \pm)\end{array}$ & $\begin{array}{l}\text { C.D. @ } \\
\text { 0.05\% }\end{array}$ & F-test & $\begin{array}{l}\text { S. Ed. } \\
( \pm)\end{array}$ & C.D. @ $0.05 \%$ \\
\hline $\begin{array}{l}\text { Due to } \\
\text { depth }\end{array}$ & $\mathrm{S}$ & 85.78907 & 0.00000001 & $\mathrm{~S}$ & 6.055716 & 0.00000002 & $\mathrm{~S}$ & 17.32161 & 0.00000000001 \\
\hline $\begin{array}{c}\text { Due to } \\
\text { site }\end{array}$ & $\mathrm{S}$ & 36.04452 & 0.0080572 & $\mathrm{~S}$ & 3.053152 & 0.0015092 & $\mathrm{~S}$ & 5.654408 & 0.000805119 \\
\hline
\end{tabular}

Table.6 Assessment of Exchangeable calcium, magnesium and available sulphur of Soil from different depth 0-15, 15-30 and 30-45 cm of village Piprola Ahmedpur, Shahjahanpur

\begin{tabular}{|c|c|c|c|c|c|c|c|c|c|}
\hline \multirow[t]{2}{*}{$\begin{array}{l}\text { Farmer } \\
\text { site }\end{array}$} & \multicolumn{3}{|c|}{$\begin{array}{l}\text { Exchangeable calcium } \\
\quad\left(\mathbf{c m o l}\left(\mathrm{p}^{+}\right) \mathrm{kg}^{-1}\right)\end{array}$} & \multicolumn{3}{|c|}{$\begin{array}{l}\text { Exchangeable magnesium } \\
\left(\mathrm{cmol}\left(\mathrm{p}^{+}\right) \mathrm{kg}^{-1}\right)\end{array}$} & \multicolumn{3}{|c|}{$\begin{array}{l}\text { Available sulphur } \\
\text { (ppm) }\end{array}$} \\
\hline & $\begin{array}{l}0-15 \\
\text { cm }\end{array}$ & $15-30 \mathrm{~cm}$ & $30-45 \mathrm{~cm}$ & $\begin{array}{c}0-15 \\
\mathrm{~cm}\end{array}$ & $15-30 \mathrm{~cm}$ & $\begin{array}{c}30-45 \\
\mathrm{~cm}\end{array}$ & $\begin{array}{c}0-15 \\
\text { cm }\end{array}$ & $15-30 \mathrm{~cm}$ & $30-45 \mathrm{~cm}$ \\
\hline $\mathbf{F}_{1} \mathbf{S}_{1}$ & 2.32 & 1.98 & 1.51 & 0.82 & 0.51 & 0.39 & 10.02 & 8.52 & 7.21 \\
\hline $\mathbf{F}_{1} \mathbf{S}_{2}$ & 1.83 & 1.45 & 1.23 & 0.67 & 0.54 & 0.47 & 9.78 & 7.91 & 6.45 \\
\hline $\mathbf{F}_{1} \mathbf{S}_{3}$ & 1.02 & 0.90 & 0.86 & 0.73 & 0.54 & 0.32 & 9.32 & 7.17 & 6.93 \\
\hline $\mathbf{F}_{2} \mathbf{S}_{1}$ & 1.23 & 1.05 & 1.01 & 0.64 & 0.59 & 0.23 & 7.21 & 6.92 & 6.03 \\
\hline $\mathbf{F}_{2} \mathbf{S}_{2}$ & 2.24 & 2.12 & 2.10 & 0.57 & 0.43 & 0.37 & 8.21 & 7.42 & 6.65 \\
\hline $\mathbf{F}_{2} \mathbf{S}_{3}$ & 1.87 & 1.48 & 1.34 & 0.43 & 0.35 & 0.30 & 8.63 & 7.68 & 6.93 \\
\hline $\mathbf{F}_{3} \mathbf{S}_{1}$ & 1.65 & 1.39 & 1.24 & 0.21 & 0.20 & 0.20 & 11.34 & 9.73 & 8.33 \\
\hline $\mathbf{F}_{3} \mathbf{S}_{2}$ & 2.57 & 1.98 & 0.97 & 0.39 & 0.25 & 0.21 & 9.21 & 8.22 & 5.34 \\
\hline $\mathbf{F}_{3} \mathbf{S}_{3}$ & 1.61 & 1.23 & 0.72 & 0.49 & 0.38 & 0.29 & 8.67 & 7.15 & 6.43 \\
\hline & $\begin{array}{c}\text { F- } \\
\text { test }\end{array}$ & S. Ed. ( \pm$)$ & $\begin{array}{l}\text { C.D. @ } \\
\text { 0.05\% }\end{array}$ & $\begin{array}{c}\text { F- } \\
\text { test }\end{array}$ & S. Ed. ( \pm$)$ & $\begin{array}{l}\text { C.D. @ } \\
\text { 0.05\% }\end{array}$ & F-test & S. Ed. ( $( \pm)$ & $\begin{array}{l}\text { C.D. @ } \\
\text { 0.05\% }\end{array}$ \\
\hline $\begin{array}{l}\text { Due to } \\
\text { depth }\end{array}$ & $\mathrm{S}$ & 0.297822 & 0.000313 & $\mathrm{~S}$ & 0.120652 & 0.00006 & S & 1.227877 & 0.0000002 \\
\hline $\begin{array}{c}\text { Due to } \\
\text { site }\end{array}$ & S & 0.407755 & 0.000142 & S & 0.127932 & 0.00043 & S & 0.871542 & 0.000222 \\
\hline
\end{tabular}


Fig.1 Graphical representation of Bulk density $\left(\mathrm{Mg} \mathrm{m}^{-3}\right)$ in different sites of village

Piprola Ahmedpur

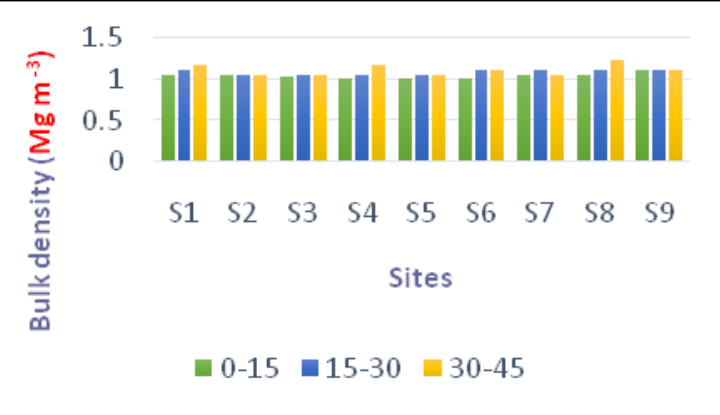

Fig.3 Graphical representation of Pore space (\%) in different sites of village Piprola Ahmedpur

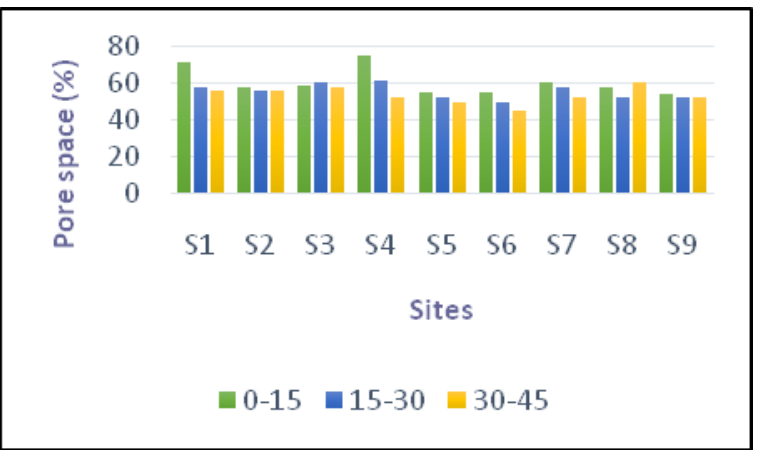

Fig.5 Graphical representation of Specific gravity in different sites of village Piprola

Ahmedpur

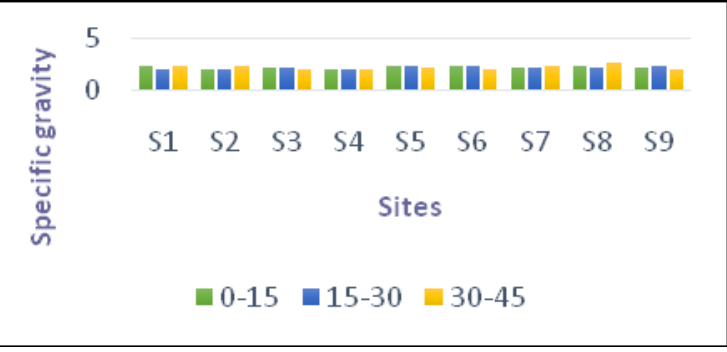

Fig.2 Graphical representation of Particle density $\left(\mathrm{Mg} \mathrm{m}^{-3}\right)$ in different sites of village

Piprola Ahmedpur

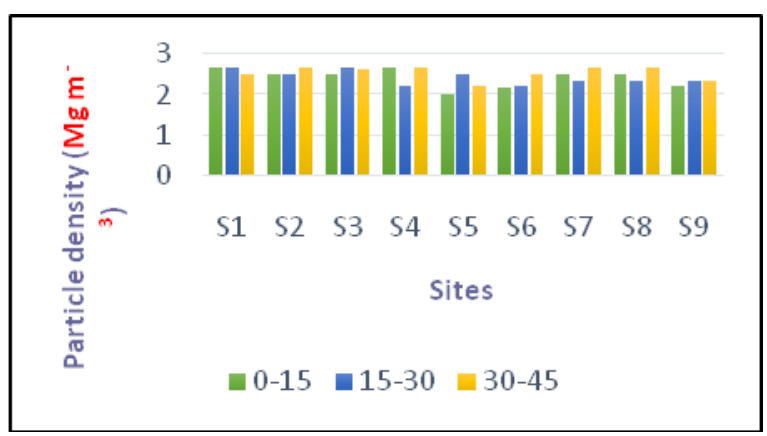

Fig.4 Graphical representation of Water holding capacity $(\%)$ in different sites of village Piprola Ahmedpur

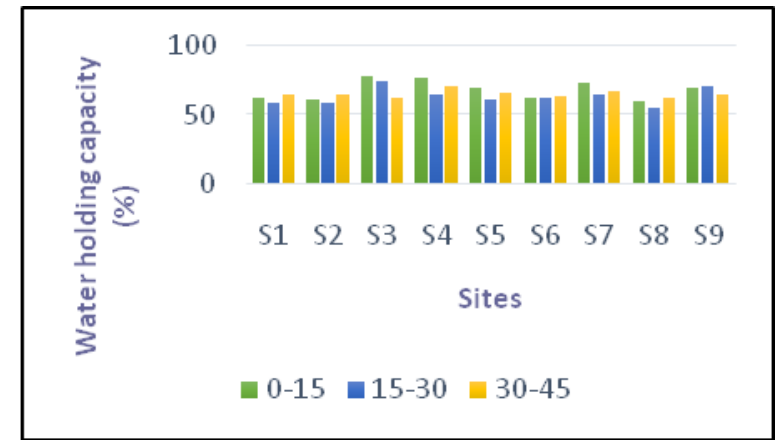

Fig.6 Graphical representation of $\mathrm{pH}$ in different sites of village Piprola Ahmedpur

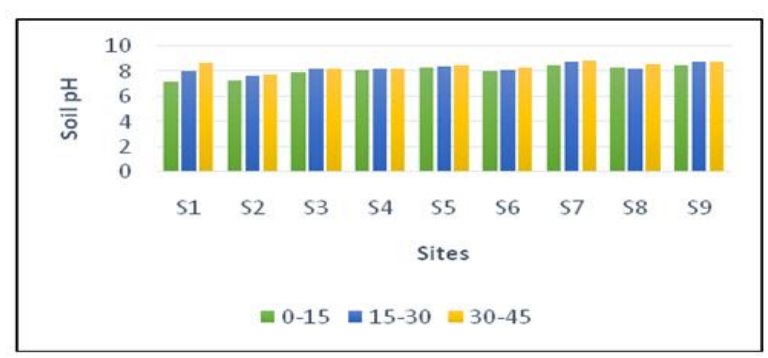


Fig.7 Graphical representation of EC (dS m ${ }^{1}$ )in different sites of village Piprola Ahmedpur

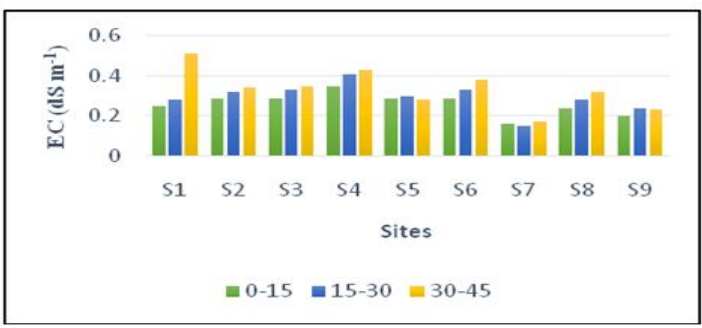

Fig.9 Graphical representation of Available $\mathrm{N}\left(\mathrm{kg} \mathrm{ha}^{-1}\right)$ in different sites of village Piprola Ahmedpur

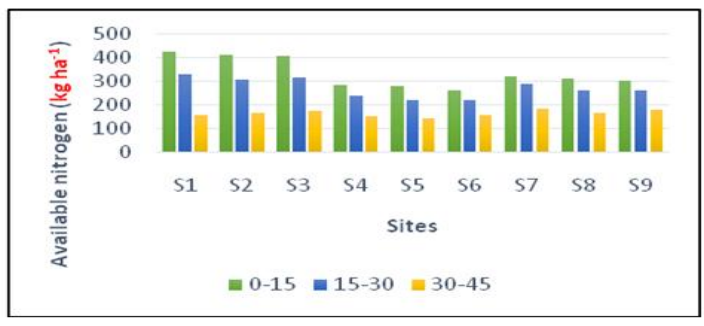

Fig.11 Graphical representation of Available $\mathrm{K}\left(\mathrm{kg} \mathrm{ha}^{-1}\right)$ in different sites of village Piprola Ahmedpur

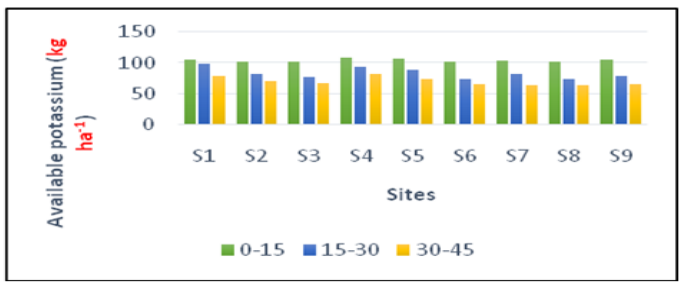

Fig.13 Graphical representation of Exchangeable Magnesium $\left(\operatorname{cmol}\left(\mathrm{p}^{+}\right) \mathrm{kg}^{-1}\right)$ in differentsites of village Piprola Ahmedpur

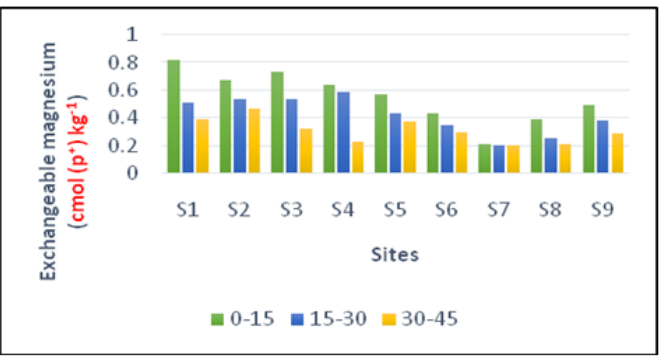

Fig.8 Graphical representation of Organic carbon (\%) in different sites of village

Piprola Ahmedpur

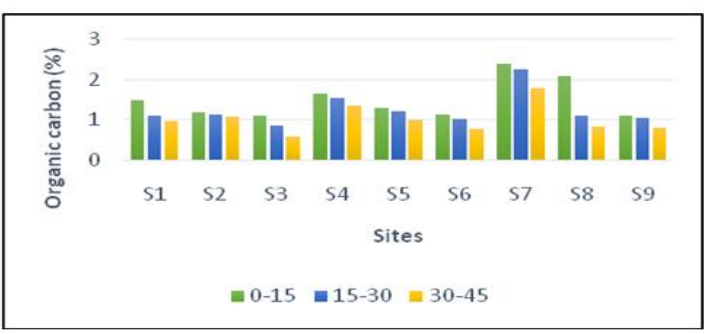

Fig.10 Graphical representation of Available $\mathrm{P}\left(\mathrm{kg} \mathrm{ha}^{-1}\right)$ in different sites of village

Piprola Ahmedpur

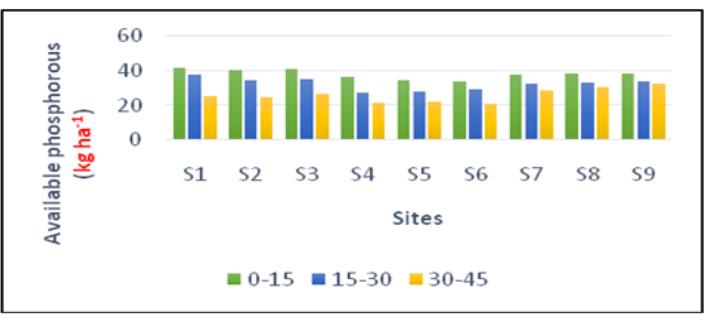

Fig.12 Graphical representation of Exchangeable Calcium $\left(\operatorname{cmol}\left(\mathrm{p}^{+}\right) \mathrm{kg}^{-1}\right)$ in differentsites of village Piprola Ahmedpur

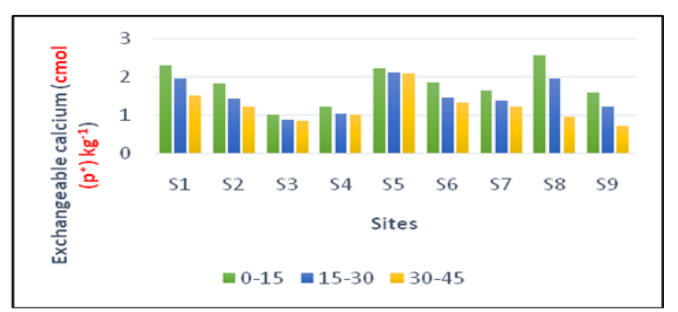

Fig.14 Graphical representation of Available sulphur (ppm) in different sites of village Piprola Ahmedpur

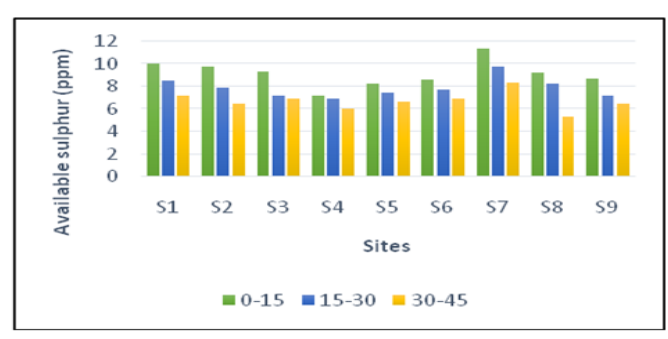


Fig.15 Soil heath card for farmer of village Piprola Ahmedpur

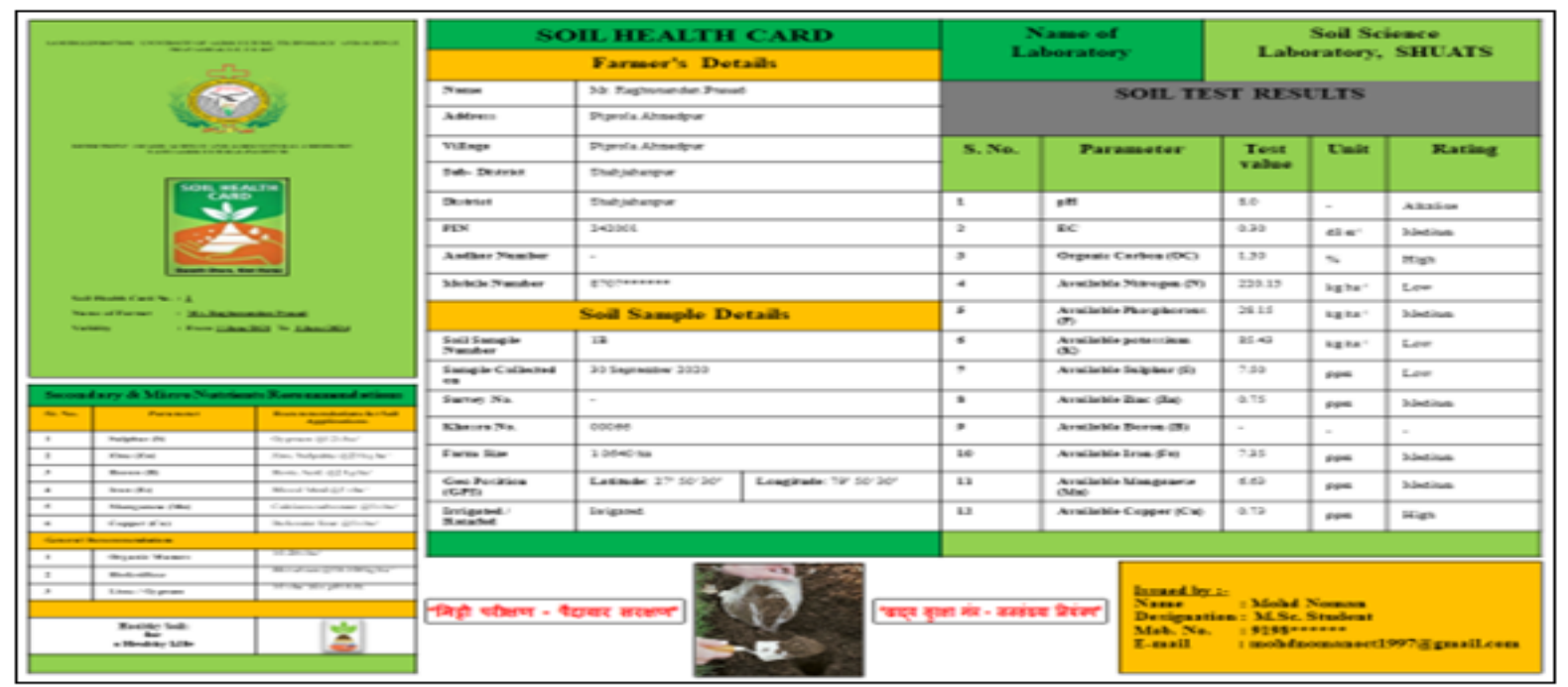

As presented in table 5 and fig. 9statistical accumulation on available nitrogen $\left(\mathrm{kg} \mathrm{ha}^{-1}\right)$ of soil in village Piprola Ahmedpur. Significant difference was found due to depth and significant difference was found due to site. The available nitrogen $\left(\mathrm{kg} \mathrm{ha}^{-1}\right)$ ranged from142.16 to $424.39\left(\mathrm{~kg} \mathrm{ha}^{-1}\right)$. The maximum value found in $\mathrm{F}_{1} \mathrm{~S}_{1}(0-15 \mathrm{~cm}$ depth) $424.39(\mathrm{~kg}$ $\mathrm{ha}^{-1}$ ) and the minimum value found in $\mathrm{F}_{2} \mathrm{~S}_{2}\left(30-45 \mathrm{~cm}\right.$ depth) $142.16\left(\mathrm{~kg} \mathrm{ha}^{-1}\right)$. The available nitrogen decreases with the increasing depth due to the fact it is positively correlated with organic matter content which decreases with depth and might be due to higher $\mathrm{pH}$ to the depth. Similar finding were reported by Ho et al., (2019) ${ }^{(8)}$. As depicted in table 5 and fig. 10statistical accumulation on available phosphorous $\left(\mathrm{kg} \mathrm{ha}^{-1}\right)$ of soil in village Piprola Ahmedpur. Significant difference was found due to depth and significant difference was found due to site. The available phosphorous $\left(\mathrm{kg} \mathrm{ha}^{-1}\right)$ ranged from 20.67 to $41.42\left(\mathrm{~kg} \mathrm{ha}^{-1}\right)$. The maximum value found in $F_{1} S_{1}(0-15 \mathrm{~cm}$ depth) $41.42(\mathrm{~kg}$ $\left.\mathrm{ha}^{-1}\right)$ and the minimum value found in $\mathrm{F}_{2} \mathrm{~S}_{3}(30-$ $45 \mathrm{~cm} \mathrm{depth)} 20.67\left(\mathrm{~kg} \mathrm{ha}^{-1}\right)$. The available phosphorous decreases with the increasing depth. Higher level of available phosphorous in surface soil could be attribute of favourable soil $\mathrm{pH}$ and organic matter content. Similar finding were reported by Wani et al., $(2017)^{(28)}$.As shown in table 5 and fig. 11statistical accumulation on available potassium $\left(\mathrm{kg} \mathrm{ha}^{-1}\right)$ of soil in village Piprola Ahmedpur. Significant difference was found due to depth and significant difference was found due to site. The available potassium $(\mathrm{kg}$ $\left.\mathrm{ha}^{-1}\right)$ ranged from 62.88 to $108.23\left(\mathrm{~kg} \mathrm{ha}^{-1}\right)$. The maximum value found in $\mathrm{F}_{2} \mathrm{~S}_{1}(0-15 \mathrm{~cm}$ depth) $108.23\left(\mathrm{~kg} \mathrm{ha}^{-1}\right)$ and the minimum value found in $\mathrm{F}_{3} \mathrm{~S}_{2}\left(30-45 \mathrm{~cm}\right.$ depth) $62.88\left(\mathrm{~kg} \mathrm{ha}^{-1}\right)$. The available potassium decreases with the increasing depth. The high content of available potassium on surface soil may be attributed to the release of labile $\mathrm{K}$ from organic residues and application of potassium fertilizers. Similar finding were reported by Singh et al., $(2020)^{(20)}$. As presented in table 6 and fig. 12statistical accumulation on exchangeable calcium $\left(\mathrm{cmol}\left(\mathrm{p}^{+}\right) \mathrm{kg}^{-1}\right)$ of soil in village Piprola Ahmedpur. Significant difference was found due to depth and significant difference was found due to site. The exchangeable calcium $\left(\mathrm{cmol}\left(\mathrm{p}^{+}\right) \mathrm{kg}^{-1}\right)$ ranged from 0.72 to $2.57\left(\mathrm{cmol}\left(\mathrm{p}^{+}\right) \mathrm{kg}^{-1}\right)$. The maximum value found in $\mathrm{F}_{3} \mathrm{~S}_{2}(0-15 \mathrm{~cm}$ depth) 
$2.57\left(\mathrm{cmol}\left(\mathrm{p}^{+}\right) \mathrm{kg}^{-1}\right)$ and the minimum value found in $\mathrm{F}_{3} \mathrm{~S}_{3}\left(30-45 \mathrm{~cm}\right.$ depth) $0.72\left(\mathrm{cmol}\left(\mathrm{p}^{+}\right)\right.$ $\left.\mathrm{kg}^{-1}\right)$. The exchangeable calcium decreases with the increasing depth due to the attribute of high $\mathrm{pH}$. Similar finding were reported by Okolo et al., $(2016)^{(15)}$. As depicted in table 6 and fig. 13statistical accumulation on exchangeable magnesium $\left(\mathrm{cmol}\left(\mathrm{p}^{+}\right) \mathrm{kg}^{-1}\right)$ of soil in village Piprola Ahmedpur. Significant difference was found due to depth and significant difference was found due to site. The exchangeable magnesium $\left(\mathrm{cmol}\left(\mathrm{p}^{+}\right) \mathrm{kg}^{-1}\right)$ ranged from0.20 to $0.82\left(\mathrm{cmol}\left(\mathrm{p}^{+}\right) \mathrm{kg}^{-1}\right)$. The maximum value found in $\mathrm{F}_{1} \mathrm{~S}_{1}(0-15 \mathrm{~cm}$ depth $)$ $0.82\left(\mathrm{cmol}\left(\mathrm{p}^{+}\right) \mathrm{kg}^{-1}\right)$ and the minimum value found in $\mathrm{F}_{3} \mathrm{~S}_{1}\left(30-45 \mathrm{~cm}\right.$ depth) $0.20\left(\mathrm{cmol}\left(\mathrm{p}^{+}\right)\right.$ $\left.\mathrm{kg}^{-1}\right)$. The exchangeable magnesium decreases with the increasing depth due to the attribute of high $\mathrm{pH}$. Similar finding were reported by Okolo et al., (2016) ${ }^{(15)}$. As shown in table 6 and fig. 14 statistical accumulation on available sulphur (ppm) of soil in village Piprola Ahmedpur. Significant difference was found due to depth and significant difference was found due to site. The available sulphur (ppm) ranged from 6.03 to 11.34 (ppm). The maximum value found in $\mathrm{F}_{3} \mathrm{~S}_{1}(0-15 \mathrm{~cm}$ depth $)$ $11.34(\mathrm{ppm})$ and the minimum value found in $\mathrm{F}_{2} \mathrm{~S}_{1}(30-45 \mathrm{~cm}$ depth) 6.03(ppm). The available sulphur decreases with the increasing depth might be due to greater plant and microbial activities and mineralization of organic matter in surface layer. Similar finding were reported by Singh et al., $(2019)^{(18)}$.

The trial that the soils of Shahjahanpur Piprola village is sandy loam having good physical conditions. It is neutral to alkaline as favourable electrical conductivity for plant growth, fertile with high organic content and low to medium of macronutrients viz. nitrogen, phosphorous and potassium. Some sites showed a deficiency in secondary nutrients i.e. calcium, magnesium and sulphur. The deficiency of the nutrients can be mitigate by the use of organic and inorganic fertilizers. It shows that the soils are good for cultivation of paddy, wheat, maize, millet, pulses, potato, sugarcane etc. Farmers are required to maintain Soil Health Card according to the guidelines of central and state government for crop cultivation and advise to adopt suitable management practices and provide proper nutrition to soil health. Time to time inventory should be maintained to overcome to the pollution effect in their respective soil.

\section{Acknowledgement}

The authors would like to thank the Hon'ble Vice Chancellor, SHUATS and HoD, Department of Soil Science and Agricultural Chemistry, Naini Agriculture Institute, Sam Higginbottom University of Agriculture Technology and Sciences for the support. The thankfulness is also extended to all professors, friends and technicians for their continuous help and support.

\section{References}

1. Bardsley $\mathrm{C}$ E and Lancaster $\mathrm{J}$ D. Determination of reserve sulphur and soluble sulphates in soil. Soil Sci. Amer. Proc.1960;24:265-268.

2. Bhattacharyya R, Ghosh B N, Mishra P K, Mandal B, Rao C S, Sarkar D et al., Soil degradation in India: challenges and potential solutions. Sustainability 2015;7:3528-3570.

3. Black C A. Methods of soil analysis. American Society of Agronomy, Madison, Wisconsin, USA, 1965;2.

4. Bouyoucos G J. The hydrometer as a new method for the mechanical analysis of soils. Soil Science 1927;23:343-353.

5. Cheng $\mathrm{K} L$ and Bray $\mathrm{R} \mathrm{H}$. Determination of Calcium and Magnesium in soil and plant material.Soil Sci.1951;72:449-458. 
6. Fisher R A. Statistical methods and scientific induction. Journal of the royal statistical society series 1960;17:69-78.

7. Harrell J B. An evaluation of soil sampling methods in support of precision agriculture in Northeastern North Carolina. 2014.

8. Ho S Y, Wasli M E B and Perumal M. Evaluation of physicochemical properties of sandy-textured soils under smallholder agricultural land use practices in Sarawak, East Malaysia. Applied and Environmental Soil Science2019.Article ID 7685451. https://doi.org/10.1155/2019/7685451

9. Idowu J, Ghimire R, Flynn R and Ganguli A. Soil Health-Importance, Assessment, and Management. Cooperative Extension Service 2019; Circular 694B. aces.nmsu.edu/pubs

10. ISRIC. Soils and Food Security. World soil information 2021;Retrieved from https://www.isric.org/utilise/globalissu es\#: :text=Land\%20degradation $\% 20 \mathrm{a}$ nd\%20its\%20negative, which\%20our\% 20life\%20depends\%20on.\&text=Glob ally\%2C\%20two\%2Dthirds\%20of $\% 20$ all,in\%20soils\%20as\%20soil\%20moist ure

11. Jackson M L. Soil Chemical Analysis. Prentice Hall of India. Private Ltd., New Delhi. 1958.

12. Khadka D, Lamichhane S, Shrestha S $\mathrm{R}$ and Pant B B. Evaluation of soil fertility status of Regional Agricultural Research Station, Tarahara, Sunsari, Nepal. Eurasian J Soil Sci 2017;6(4): 295-306.

13. Mallick S R, Proshad R, Islam M S, Sayeed A, Uddin M, Gao J and Zhang, D. Heavy metals toxicity of surface soils near industrial vicinity: A study on soil contamination in Bangladesh. Arch. Agr. Environ. Sci. 2019;4(4):356-368.
14. Muthuvel P, Udayasoorian C, Natesan $\mathrm{R}$ and Ramaswami P R. Introduction to Soil Analysis. Tamil Nadu Agricultural University, Coimbatore. 1992.

15. Okolo V N, Olowolafe E A, Akawu I and Okoduwa $\mathrm{S} I \mathrm{R}$. Effects of industrial effluents on soil resource in challawa industrial area, Kano, Nigeria. Journal of Global Ecology and Environment 2016;5(1):1-10.

16. Olsen S R, Cole C V, Watnahe F S and Dean L A. Estimation of available phosphorus in soils by extraction with sodium bicarbonate U.S. Deptt. Agr. Circ. 1954;939.

17. Ranjan V. District ground water brochure of Shahjahanpur district, U.P.2013.

18. Singh A K, Singh A K and Singh A K. Study of available potassium by different extracting reagents and fertility status of soils of Bairia subdivision of Ballia, U.P. Asian Journal of Science and Technology2019;9(04):9584-9586.

19. Singh B and Sharma K N. Depth wise distribution of soil organic carbon and nutrients under some tree species after seventeen years of plantation. Journal of the Indian Society of Soil Science2012;60(3):198-203.

20. Singh D, Singh A K, Singh A K and Gupta S K. Characterization of rice growing soil of Nagara block of Ballia District (U.P.), India. Int. J. Curr. Microbiol. App. Sci. 2020;9(04):575581 .

21. Subbiah B V and Asija C L. A rapid procedure for the estimation of available nitrogen in soils. Current Sci. 1956;25: 259-260.

22. Sujatha K N, Kavya G, Manasa P and Divya K. Assessment of soil properties to improve water holding capacity in soils. International Research Journal of 
Engineering and Technology (IRJET)2016;3(3):1777-1783.

23. Supriya. India: Soil Types, Problems $\&$

Conservation.2021.http://bhuvankosh.c 0

24. Tale K S and Ingole S. A Review on role of physico-chemical properties in soil quality. Chem Sci Rev Lett 2015;4(13):57-66.

25. Toth S J and Prince A L. Estimation of cation exchange capacity and exchangeable $\mathrm{Ca}, \mathrm{K}$ and $\mathrm{Na}$ content of soil by flame photometer technique. Soil Sci. 1949;67:439-445.

26. Verma C, Lal A, David A D M and Rao P S. Determination of physicochemical properties in soil samples of Prayagraj (Allahabad) District, Uttar Pradesh, India. Asian Journal of Applied Chemistry Research2019;4(2):1-8.
27. Walkley A. Critical examination of rapid method for determining organic carbon in soils, effect of variation in digestion conditions and of inorganic soil constituents, Soil Sci.1947;632: 251.

28. Wani S A, Najar G R, Padder B A, Akhter $\mathrm{F}$ and Chand $\mathrm{S}$. Altitudinal and depth-wise variation of soil physicochemical properties and available nutrients of pear orchards in Jammu \& Kashmir, India. Chem. Sci. Rev. Lett. 2017;6(23):1638-1645.

29. Wilcox L V. Electrical conductivity, Amer. Water Works Assoc. J 1950;42:775-776.

30. Yuvarani R, Swarrop N, Rao P S and Thomas $T$. Impact of tannery effluent on physical properties of soils of Dindigul district of Tamil Nadu state. International Journal of Chemical Studies 2019;7(3):420-422.

\section{How to cite this article:}

Mohd Noman, Arun Alfred David, Tarence Thomas, Narendra Swaroop and Amreen Hasan. 2021. Assessment of Physico-chemical Properties of Soil in Dadrol Block, Shahjahanpur District, Uttar Pradesh, India. Int.J.Curr.Microbiol.App.Sci. 10(07): 30-42. doi: https://doi.org/10.20546/ijcmas.2021.1007.004 